\title{
A Note on Optimality Conditions for DC Programs Involving Composite Functions
}

\author{
Xiang-Kai Sun ${ }^{1,2}$ and Hong-Yong $\mathrm{Fu}^{3}$ \\ ${ }^{1}$ College of Mathematics and Statistics, Chongqing Technology and Business University, Chongqing 400067, China \\ ${ }^{2}$ College of Automation, Chongqing University, Chongqing 400030, China \\ ${ }^{3}$ School of Management, Southwest University of Political Science and Law, Chongqing 401120, China
}

Correspondence should be addressed to Hong-Yong Fu; fuhongyong.cqu@gmail.com

Received 23 April 2014; Accepted 22 May 2014; Published 29 May 2014

Academic Editor: Chong Li

Copyright (c) 2014 X.-K. Sun and H.-Y. Fu. This is an open access article distributed under the Creative Commons Attribution License, which permits unrestricted use, distribution, and reproduction in any medium, provided the original work is properly cited.

By using the formula of the $\varepsilon$-subdifferential for the sum of a convex function with a composition of convex functions, some necessary and sufficient optimality conditions for a DC programming problem involving a composite function are obtained. As applications, a composed convex optimization problem, a DC optimization problem, and a convex optimization problem with a linear operator are examined at the end of this paper.

\section{Introduction}

Let $X$ and $Y$ be two real locally convex Hausdorff topology vector spaces with their dual spaces $X^{*}$ and $Y^{*}$, endowed with the weak ${ }^{*}$ topologies $w\left(X^{*}, X\right)$ and $w\left(Y^{*}, Y\right)$, respectively. Let $K \subseteq Y$ be a nonempty closed convex cone which defined the partial order " $\leq_{K}$ " of $Y$; namely,

$$
y_{1} \leq_{K} y_{2} \Longleftrightarrow y_{2}-y_{1} \in K, \quad \text { for any } y_{1}, y_{2} \in Y \text {. }
$$

We attach an element $\infty_{Y} \notin Y$ which is the greatest element with respect to " $\leq_{K}$ " and let $Y^{*}=Y \cup\left\{\infty_{Y}\right\}$. Then, for any $y \in$ $Y^{*}$, one has $y \leq_{K} \infty_{Y}$ and we define the following operations on $Y^{*}$ :

$$
\begin{array}{r}
y+\left(\infty_{Y}\right)=\left(\infty_{Y}\right)+y=\infty_{Y}, \quad t\left(\infty_{Y}\right)=\infty_{Y}, \\
\text { for any } y \in Y, t \geq 0 .
\end{array}
$$

Let $f_{1}, f_{2}: X \rightarrow \overline{\mathbb{R}}:=\overline{\mathbb{R}} \cup\{+\infty\}$ and $g: Y \rightarrow \overline{\mathbb{R}}$ be proper, convex, and lower semicontinuous functions, and let $h$ : $X \rightarrow Y^{\bullet}$ be a proper, $K$-convex, and star $K$-lower semicontinuous function such that $h\left(\operatorname{dom} f_{1} \cap \operatorname{dom} f_{2}\right) \cap \operatorname{dom} g \neq \emptyset$.
Moreover, we assume that $g$ is a $K$-increasing function; that is,

for any $x, y \in Y$ such that $x \leq_{K} y$, we have $g(x) \leq g(y)$.

In this paper, we deal with a new class of DC programming involving a composite function given in the following form:

$$
\inf _{x \in X}\left\{f_{1}(x)-f_{2}(x)+g \circ h(x)\right\} .
$$

The problem $(P)$ is very general in the sense that it includes, as particular cases, many different problems as, for example, a composed convex optimization problem, a DC optimization problem, and a convex optimization problem with a linear operator; see [1-12] and the references therein. The interest of such a general problem is that it unifies all these particular problems in a convenient way. Moreover, many results obtained for one of these problems can be extended with suitable modifications to the problem $(P)$.

Recently, optimality conditions for global or local minimizers of some special kinds of the problem $(P)$ have been studied by many researchers; see [13-25] and the references therein. Here, we specially mention the works on optimality defined via subdifferential calculus due to $[18,24,25]$. By 
using a formula for the $\varepsilon$-subdifferential of the sum of a convex function with a composition of convex functions, Boţ et al. [18] have obtained necessary and sufficient conditions for the $\varepsilon$-optimal solutions of composed convex optimization problems. By using some suitable conditions and the notions of strong subdifferential and epsilon-subdifferential, Guo and $\mathrm{Li}$ [24] obtained necessary and sufficient optimality conditions for an epsilon-weak Pareto minimal point and an epsilon-proper Pareto minimal point of a DC vector optimization problem. Fang and Zhao [25] introduced the local and global KKT type conditions for a DC optimization problem. Then, by using properties of the subdifferentials of the involved functions, they obtained some sufficient and/or necessary conditions for these two types of optimality conditions. The purpose of this paper is to establish optimality conditions for this optimization problem $(P)$. To do that, by using the properties of the epigraph of the conjugate functions, we first introduce some closedness conditions and investigate some characterizations of these closedness conditions via the formula of the $\varepsilon$-subdifferential. Then, we obtain some necessary and sufficient optimality conditions. Moreover, at the end of this paper, we examine a composed convex optimization problem, a DC optimization problem, and a convex optimization problem with a linear operator.

The paper is organized as follows. In Section 2, we recall some notions and give some preliminary results. In Section 3, we obtain some optimality conditions for the problem $(P)$ in terms of the subdifferentials and the $\varepsilon$-subdifferentials of the functions. In Section 4, we give some special cases of our general results, which have been treated in the previous papers.

\section{Mathematical Preliminaries}

Throughout this paper, let $X$ and $Y$ be two real locally convex Hausdorff topology vector spaces. Let $D$ be a set in $X$; the interior (resp., closure, convex hull, and convex cone hull) of $D$ is denoted by int $D$ (resp., $\operatorname{cl} D, \operatorname{co} D$, and cone $D$ ). Thus, if $W \subseteq X^{*}$, then $\mathrm{cl} W$ denotes the weak ${ }^{*}$ closure of $W$. We shall adopt the convention that cone $D=\{0\}$ when $D$ is an empty set. Let $D^{*}=\left\{x^{*} \in X^{*}:\left\langle x^{*}, x\right\rangle \geq 0, \forall x \in X\right\}$ be the dual cone of $D$. The indicator function $\delta_{D}: X \rightarrow \overline{\mathbb{R}}$ of $X$ is defined by

$$
\delta_{D}(x)= \begin{cases}0, & \text { if } x \in D \\ +\infty, & \text { if } x \notin D\end{cases}
$$

The support function $\sigma_{D}: X^{*} \rightarrow \overline{\mathbb{R}}$ of $D$ is defined by

$$
\sigma_{D}\left(x^{*}\right)=\sup _{x \in D}\left\langle x^{*}, x\right\rangle .
$$

Let $f: X \rightarrow \overline{\mathbb{R}}$ be an extended real valued function. The effective domain and the epigraph are defined by

$$
\begin{aligned}
\operatorname{dom} f & =\{x \in X: f(x)<+\infty\}, \\
\text { epi } f & =\{(x, r) \in X \times \mathbb{R}: f(x) \leq r\},
\end{aligned}
$$

respectively. $f$ is said to be proper if and only if its effective domain is nonempty and $f(x)>-\infty$. The conjugate function $f^{*}: X^{*} \rightarrow \overline{\mathbb{R}}$ of $f$ is defined by

$$
f^{*}\left(x^{*}\right)=\sup _{x \in X}\left\{\left\langle x^{*}, x\right\rangle-f(x)\right\} .
$$

Let $\bar{x} \in \operatorname{dom} f$. For any $\varepsilon \geq 0$, the $\varepsilon$-subdifferential of $f$ at $\bar{x}$ is the convex set defined by

$$
\begin{aligned}
& \partial_{\varepsilon} f(\bar{x}) \\
& =\left\{x^{*} \in X^{*}: f(x) \geq f(\bar{x})+\left\langle x^{*}, x-\bar{x}\right\rangle-\varepsilon, \forall x \in X\right\} .
\end{aligned}
$$

When $\bar{x} \notin \operatorname{dom} f$, we define that $\partial_{\varepsilon} f(\bar{x})=\emptyset$. If $\varepsilon=0$, the set $\partial f(\bar{x}):=\partial_{0} f(\bar{x})$ is the classical subdifferential of convex analysis. It is easy to prove that, for any $\bar{x} \in \operatorname{dom} f$ and $x^{*} \in$ $X^{*}$,

$$
f(\bar{x})+f^{*}\left(x^{*}\right) \leq\left\langle x^{*}, \bar{x}\right\rangle+\varepsilon \Longleftrightarrow x^{*} \in \partial_{\varepsilon} f(\bar{x}) .
$$

Let $E$ be a convex set of $X$. The $\varepsilon$-normal cone to $E$ at a point $\bar{x} \in E$ is defined by $N_{\varepsilon}(E, \bar{x})=\left\{x^{*} \in X^{*}:\left\langle x^{*}, x-\right.\right.$ $\bar{x}\rangle \leq \varepsilon$, for any $x \in E\}$. If $\varepsilon=0, N_{0}(E, \bar{x})$ is the normal cone $N(E, \bar{x})$ of convex analysis. Moreover, it is easy to see that $N_{\varepsilon}(E, \bar{x})=\partial_{\varepsilon} \delta_{E}(\bar{x})$.

Let $A: X \rightarrow Y$ be a linear continuous mapping. The adjoint mapping $A^{*}: Y^{*} \rightarrow X^{*}$ of $A$ is defined by

$$
\left\langle A^{*} y^{*}, x\right\rangle=\left\langle y^{*}, A x\right\rangle, \quad \text { for any }\left(x, y^{*}\right) \in X \times Y^{*} \text {. }
$$

The infimal function $A f: Y \rightarrow \overline{\mathbb{R}}$ of $f$ through $A$ is defined by

$$
A f(y)=\inf \{f(x): x \in X, A x=y\}, \quad \text { for any } y \in Y .
$$

Let $h: X \rightarrow Y^{\bullet}$ be an extended vector valued function. The domain and the $K$-epigraph of $h$ are defined by

$$
\begin{aligned}
\operatorname{dom} h & =\{x \in X: h(x) \in Y\}, \\
\operatorname{epi}_{K} h & =\{(x, y) \in X \times Y: y \in h(x)+K\},
\end{aligned}
$$

respectively. $h$ is said to be proper if and only if dom $h \neq \emptyset$. $h$ is said to be a $K$-convex function if and only if, for any $x, y \in X$ and $t \in[0,1]$, we have

$$
h(t x+(1-t) y) \leq_{K} t h(x)+(1-t) h(y) .
$$

For any subset $W \subseteq Y$, we denote

$$
\begin{aligned}
& h^{-1}(W) \\
& =\{x \in X: \text { there exists } y \in W \text { such that } h(x)=y\} .
\end{aligned}
$$

Moreover, let $\lambda \in K^{*}$. The function $(\lambda h): X \rightarrow \overline{\mathbb{R}}$ is defined by

$$
(\lambda h)(x)= \begin{cases}\langle\lambda, h(x)\rangle, & \text { if } x \in \operatorname{dom} h, \\ +\infty, & \text { otherwise. }\end{cases}
$$

We say that $h$ is star $K$-lower semicontinuous if and only if $(\lambda h)$ is lower semicontinuous, for any $\lambda \in K^{*}$.

Now, let us recall the following result which will be used in the following section. 
Lemma 1 (see [26]). Let $f_{1}, f_{2}: X \rightarrow \overline{\mathbb{R}}$ be proper, convex, and lower semicontinuous functions. Then

(i) $\bar{x}$ is a global optimal solution of $\inf _{x \in X}\left\{f_{1}(x)-f_{2}(x)\right\}$ if and only if, for any $\varepsilon \geq 0, \partial_{\varepsilon} f_{2}(\bar{x}) \subset \partial_{\varepsilon} f_{1}(\bar{x})$.

(ii) If $\bar{x}$ is a local optimal solution of $\inf _{x \in X}\left\{f_{1}(x)-f_{2}(x)\right\}$, then $\partial f_{2}(\bar{x}) \subset \partial f_{1}(\bar{x})$.

\section{Optimality Conditions for $(P)$}

In this section, we will employ the closedness qualification condition to derive necessary optimality conditions as well as necessary and sufficient optimality conditions for local and global minimizers in DC programs of type $(P)$. Now, we first recall the closedness qualification condition (CQC).

Definition 2 (see [3]). The problem $(P)$ is said to satisfy the closedness qualification condition (CQC) if the set

$$
\text { epi } f_{1}^{*}+\bigcup_{\lambda \in \operatorname{dom} g^{*}}\left(\operatorname{epi}(\lambda h)^{*}+\left(0, g^{*}(\lambda)\right)\right)
$$

is weak ${ }^{*}$ closed in the space $X^{*} \times \mathbb{R}$.

The next lemma provides several characterizations of the closedness qualification condition (CQC). Moreover, the condition will be crucial in the sequel and it also deserves some attention for its independent interest.

Lemma 3 (see [3]). The closedness qualification condition (CQC) holds if and only if, for any $x \in \operatorname{dom} f_{1} \cap h^{-1}$ (dom $g$ ) and any $\varepsilon \geq 0$,

$$
\begin{aligned}
& \partial_{\varepsilon}\left(f_{1}+g \circ h\right)(x) \\
& =\bigcup_{\substack{\varepsilon_{1}, \varepsilon_{2}, \varepsilon_{3} \geq 0, \varepsilon_{1}+\varepsilon_{2}+\varepsilon_{3}=\varepsilon}}\left\{\partial_{\varepsilon_{1}} f_{1}(\bar{x})+\partial_{\varepsilon_{2}}(\lambda h)(x): \lambda \in K^{*} \cap \partial_{\varepsilon_{3}} g(h(x))\right\} .
\end{aligned}
$$

Taking $\varepsilon=0$ in Lemma 3, we can easily obtain the following subdifferential sum rule.

Corollary 4. If the closedness qualification condition (CQC) holds, then, for any $x \in \operatorname{dom} f_{1} \cap h^{-1}(\operatorname{dom} g)$,

$$
\partial\left(f_{1}+g \circ h\right)(x)=\partial f_{1}(\bar{x})+\bigcup_{\lambda \in K^{*} \cap \partial g(h(x))} \partial(\lambda h)(x) .
$$

Now, by using the closedness qualification condition and the $\varepsilon$-subdifferential sum rule, we establish necessary and sufficient optimality conditions for global optimal solution of $(P)$.

Theorem 5. Let $\bar{x} \in \operatorname{dom} f_{1} \cap \operatorname{dom} f_{2} \cap h^{-1}(\operatorname{dom} g)$. Suppose that the closedness qualification condition (CQC) holds. Then, $\bar{x}$ is a global optimal solution of $(P)$ if and only if, for any $\varepsilon \geq 0$, there exist $\varepsilon_{1}, \varepsilon_{2}, \varepsilon_{3} \geq 0$ and $\lambda \in K^{*} \cap \partial_{\varepsilon_{3}} g(h(x))$ such that

$$
\begin{gathered}
\partial_{\varepsilon} f_{2}(\bar{x}) \subseteq \partial_{\varepsilon_{1}} f_{1}(\bar{x})+\partial_{\varepsilon_{2}}(\lambda h)(\bar{x}), \\
\varepsilon_{1}+\varepsilon_{2}+\varepsilon_{3}=\varepsilon
\end{gathered}
$$

Proof. It is clear that $(P)$ can be rewritten as

$$
\inf _{x \in X}\left\{\left(f_{1}+g \circ h\right)(x)-f_{2}(x)\right\} .
$$

Then, by Lemma $1, \bar{x}$ is a global optimal solution of $(P)$ if and only if, for any $\varepsilon \geq 0$,

$$
\partial_{\varepsilon} f_{2}(\bar{x}) \subset \partial_{\varepsilon}\left(f_{1}+g \circ h\right)(\bar{x}) .
$$

Moreover, by Lemma 3, this is further equivalent to

$$
\begin{aligned}
& \partial_{\varepsilon} f_{2}(\bar{x}) \\
& \subseteq \bigcup_{\substack{\varepsilon_{1}, \varepsilon_{2}, \varepsilon_{3} \geq 0, \varepsilon_{1}+\varepsilon_{2}+\varepsilon_{3}=\varepsilon}}\left\{\partial_{\varepsilon_{1}} f_{1}(\bar{x})+\partial_{\varepsilon_{2}}(\lambda h)(x): \lambda \in K^{*} \cap \partial_{\varepsilon_{3}} g(h(x))\right\} .
\end{aligned}
$$

This means that, for any $\varepsilon \geq 0$, there exist $\varepsilon_{1}, \varepsilon_{2}, \varepsilon_{3} \geq 0$ and $\lambda \in K^{*} \cap \partial_{\varepsilon_{3}} g(h(x))$ such that $\varepsilon_{1}+\varepsilon_{2}+\varepsilon_{3}=\varepsilon$ and $\partial_{\varepsilon} f_{2}(\bar{x}) \subset$ $\partial_{\varepsilon_{1}} f_{1}(\bar{x})+\partial_{\varepsilon_{2}}(\lambda h)(\bar{x})$. This completes the proof.

The following result establishes necessary optimality conditions for local optimal solution of $(P)$.

Corollary 6. Let $\bar{x} \in \operatorname{dom} f_{1} \cap \operatorname{dom} f_{2} \cap h^{-1}(\operatorname{dom} g)$. Suppose that the closedness qualification condition (CQC) holds. If $\bar{x}$ is a local optimal solution of $(P)$, then there exists $\lambda \in K^{*} \cap$ $\partial g(h(x))$ such that

$$
\partial f_{2}(\bar{x}) \subseteq \partial f_{1}(\bar{x})+\partial(\lambda h)(\bar{x}) .
$$

Proof. If $\bar{x}$ is a local optimal solution of $(P)$, then, by Lemma 1,

$$
\partial f_{2}(\bar{x}) \subset \partial\left(f_{1}+g \circ h\right)(\bar{x}) .
$$

By Corollary 4,

$$
\partial f_{2}(\bar{x}) \subseteq \partial f_{1}(\bar{x})+\bigcup_{\lambda \in K^{*} \cap \partial g(h(x))} \partial(\lambda h)(x),
$$

which means that there exists $\lambda \in K^{*} \cap \partial g(h(x))$ such that

$$
\partial f_{2}(\bar{x}) \subseteq \partial f_{1}(\bar{x})+\partial(\lambda h)(\bar{x}) .
$$

This completes the proof.

\section{The Special Cases}

In this section, we will give some special cases of our general results, which have been treated in the previous papers.

4.1. A Composed Convex Optimization Problem. When $f_{2}(x)=0,(P)$ becomes the following composed convex optimization problem:

$$
\inf _{x \in X}\left\{f_{1}(x)+g \circ h(x)\right\} .
$$

As some consequences of the results which have been treated in Section 3, we obtain the following results for $\left(P_{1}\right)$ which was established in [18]. 
Theorem 7. Let $\bar{x} \in \operatorname{dom} f_{1} \cap h^{-1}(\operatorname{dom} g)$. Suppose that the closedness qualification condition (CQC) holds. Then, $\bar{x}$ is an $\varepsilon$-optimal solution of $\left(P_{1}\right)$ if and only if, for any $\varepsilon \geq 0$, there exist $\varepsilon_{1}, \varepsilon_{2}, \varepsilon_{3} \geq 0$ and $\lambda \in K^{*} \cap \partial_{\varepsilon_{3}} g(h(\bar{x}))$ such that

$$
\begin{gathered}
0 \in \partial_{\varepsilon_{1}} f_{1}(\bar{x})+\partial_{\varepsilon_{2}}(\lambda h)(\bar{x}), \\
\varepsilon_{1}+\varepsilon_{2}+\varepsilon_{3}=\varepsilon .
\end{gathered}
$$

Corollary 8. Let $\bar{x} \in \operatorname{dom} f_{1} \cap h^{-1}(\operatorname{dom} g)$. Suppose that the closedness qualification condition (CQC) holds. Then, $\bar{x}$ is a global optimal solution of $(P)$ if and only if there exists $\lambda \in$ $K^{*} \cap \partial g(h(\bar{x}))$ such that

$$
0 \in \partial f_{1}(\bar{x})+\partial(\lambda h)(\bar{x})
$$

4.2. A Constrained DC Optimization Problem. In this subsection, we consider the following DC optimization problem:

$$
\inf _{\substack{x \in X, h(x) \in-K}}\left\{f_{1}(x)-f_{2}(x)\right\}
$$

Let $g=\delta_{\{-K\}}$. Obviously, $g$ is a proper, convex, lower semicontinuous, and $K$-increasing function. Then $\left(P_{2}\right)$ can be seen as a particular case of the problem $(P)$, since it can be rewritten as

$$
\inf _{x \in X}\left\{f_{1}(x)-f_{2}(x)+\delta_{-K} \circ h\right\} .
$$

Since $g^{*}=\delta_{K^{*}}$, we obtain that dom $g^{*}=K^{*}$. Then, condition (CQC) becomes

$$
\begin{aligned}
& \text { epi } f_{1}^{*} \\
& +\bigcup_{\lambda \in K^{*}} \text { epi }(\lambda h)^{*} \text { is weak }{ }^{*} \text { closed in the space } X^{*} \times \mathbb{R} \text {. }
\end{aligned}
$$

$(\mathrm{CQC})_{1}$

Moreover, by (10), we obtain that, for any $y \in-K, \lambda \epsilon$ $\partial_{\varepsilon} \delta_{\{-K\}}(y)$ if and only if $\lambda \in K^{*}$ and $0 \leq\langle\lambda, y\rangle+\varepsilon$. Then, by Lemma 3 , we get the following result.

Lemma 9. The closedness qualification condition (CQC) holds if and only if, for any $x \in \operatorname{dom} f_{1} \cap h^{-1}(-K)$ and any $\varepsilon \geq 0$,

$$
\begin{gathered}
\partial_{\varepsilon}\left(f_{1}+\delta_{-K} \circ h\right)(x) \\
=\bigcup_{\substack{\varepsilon_{1}, \varepsilon_{2}, \varepsilon_{3} \geq 0, \varepsilon_{1}+\varepsilon_{2}+\varepsilon_{3}=\varepsilon}}\left\{\partial_{\varepsilon_{1}} f_{1}(\bar{x})+\partial_{\varepsilon_{2}}(\lambda h)(x): \lambda \in K^{*},\right. \\
\left.0 \leq\langle\lambda, h(x)\rangle+\varepsilon_{3}\right\} .
\end{gathered}
$$

Taking $\varepsilon=0$ in Lemma 9, we can easily obtain the following subdifferential sum rule.

Corollary 10. If the closedness qualification condition (CQC) holds, then, for any $x \in \operatorname{dom} f_{1} \cap h^{-1}(-K)$,

$$
\partial\left(f_{1}+\delta_{-K} \circ h\right)(x)=\partial f_{1}(\bar{x})+\bigcup_{\lambda \in K^{*}} \partial(\lambda h)(x) .
$$

Similarly, by using Lemma 9 and Corollary 10, we obtain the following results.

Theorem 11. Let $\bar{x} \in \operatorname{dom} f_{1} \cap \operatorname{dom} f_{2} \cap h^{-1}(-K)$. Suppose that the closedness qualification condition (CQC) $)_{1}$ holds. Then, $\bar{x}$ is a global optimal solution of $\left(P_{2}\right)$ if and only if, for any $\varepsilon \geq 0$, there exist $\varepsilon_{1}, \varepsilon_{2}, \varepsilon_{3} \geq 0, \lambda \in K^{*}$ and $0 \leq\langle\lambda, h(x)\rangle+\varepsilon_{3}$ such that

$$
\begin{gathered}
\partial_{\varepsilon} f_{2}(\bar{x}) \subseteq \partial_{\varepsilon_{1}} f_{1}(\bar{x})+\partial_{\varepsilon_{2}}(\lambda h)(\bar{x}), \\
\varepsilon_{1}+\varepsilon_{2}+\varepsilon_{3}=\varepsilon
\end{gathered}
$$

Corollary 12. Let $\bar{x} \in \operatorname{dom} f_{1} \cap \operatorname{dom} f_{2} \cap h^{-1}(-K)$. Suppose that the closedness qualification condition (CQC) $)_{1}$ holds. If $\bar{x}$ is a local optimal solution of $\left(P_{2}\right)$ then there exists $\lambda \in K^{*}$ such that

$$
\partial f_{2}(\bar{x}) \subseteq \partial f_{1}(\bar{x})+\partial(\lambda h)(\bar{x})
$$

4.3. A Convex Optimization Problem with a Linear Operator. Let $f_{2} \equiv 0$ and $h(x)=A x$, for any $x \in X$, where $A: X \rightarrow Y$ is a linear continuous mapping. Taking $K=\{0\}$, one has that $h$ is a $K$-convex function and $K^{*}=Y^{*}$. So, the problem $(P)$ becomes

$$
\inf _{x \in X}\{f(x)+g(A(x))\} .
$$

Since

$$
(\lambda h)^{*}(p)= \begin{cases}0, & \text { if } A^{*} \lambda=p, \\ +\infty, & \text { otherwise }\end{cases}
$$

we get

$$
\operatorname{epi}(\lambda h)^{*}=\left\{(p, r) \in X^{*} \times \mathbb{R}: p=A^{*} \lambda, r \geq 0\right\} .
$$

Then

$$
\begin{gathered}
\text { epi } f^{*}+\bigcup_{\lambda \in \operatorname{dom} g^{*}}\left(\operatorname{epi}(\lambda h)^{*}+\left(0, g^{*}(\lambda)\right)\right) \\
=\text { epi } f^{*}+\left(A^{*} \times \mathrm{id}_{\mathbb{R}}\right) \text { epi } g^{*}
\end{gathered}
$$

Thus, the condition (CQC) becomes in this special case epi $f^{*}$ $+\left(A^{*} \times \mathrm{id}_{\mathbb{R}}\right)$ epi $g^{*}$ is weak ${ }^{*}$ closed in the space $X^{*} \times \mathbb{R}$.

Moreover, for any $\varepsilon \geq 0$, it is easy to see that $\partial_{\varepsilon}(\lambda h)(x)=A^{*} \lambda$. Then, by Lemma 3, we get the following result.

Lemma 13. The closedness qualification condition $\left(R C_{A}\right)$ holds if and only if, for any $x \in \operatorname{dom} f_{1} \cap A^{-1}(\operatorname{dom}(g))$ and any $\varepsilon \geq 0$,

$$
\partial_{\varepsilon}\left(f_{1}+g \circ A\right)(x)=\bigcup_{\substack{\varepsilon_{1}, \varepsilon_{3} \geq 0, \varepsilon_{1}+\varepsilon_{3}=\varepsilon}}\left\{\partial_{\varepsilon_{1}} f_{1}(\bar{x})+A^{*} \partial_{\varepsilon_{3}} g(A x)\right\} .
$$


Taking $\varepsilon=0$ in Lemma 13, we can easily obtain the following subdifferential sum rule.

Corollary 14. If the closedness qualification condition $\left(R C_{A}\right)$ holds, then, for any $x \in \operatorname{dom} f_{1} \cap A^{-1}(\operatorname{dom}(g))$,

$$
\partial\left(f_{1}+g \circ A\right)(x)=\partial f_{1}(\bar{x})+A^{*} \partial g(A x) .
$$

Similarly, by using Lemma 13 and Corollary 14, we obtain the following results.

Theorem 15. Let $\bar{x} \in \operatorname{dom} f_{1} \cap A^{-1}(\operatorname{dom}(g))$. Suppose that the closedness qualification condition $\left(R C_{A}\right)$ holds. Then, $\bar{x}$ is a $\varepsilon$-optimal solution of $\left(P_{3}\right)$ if and only if, for any $\varepsilon \geq 0$, there exist $\varepsilon_{1}, \varepsilon_{3} \geq 0$, such that

$$
\begin{gathered}
0 \in \partial_{\varepsilon_{1}} f_{1}(\bar{x})+A^{*} \partial_{\varepsilon_{3}} g(A x), \\
\varepsilon_{1}+\varepsilon_{3}=\varepsilon
\end{gathered}
$$

Corollary 16. Let $\bar{x} \in \operatorname{dom} f_{1} \cap A^{-1}(\operatorname{dom}(g))$. Suppose that the closedness qualification condition $\left(R C_{A}\right)$ holds. Then, $\bar{x}$ is a global optimal solution of $\left(P_{3}\right)$ if and only if there exists $\lambda \in K^{*}$ such that

$$
0 \in \partial f_{1}(\bar{x})+A^{*} \partial g(A x)
$$

\section{Conflict of Interests}

The authors declare that there is no conflict of interests regarding the publication of this paper.

\section{Acknowledgments}

This research was partially supported by the National Natural Science Foundation of China (Grant no. 11301570), the Basic and Advanced Research Project of CQ CSTC (Grant no. cstc2013jcyjA00003), and the China Postdoctoral Science Foundation funded project (Grant no. 2013M540697).

\section{References}

[1] R. Horst and N. V. Thoai, "DC programming: overview," Journal of Optimization Theory and Applications, vol. 103, no. 1, pp. 1-43, 1999.

[2] M. Laghdir, "Optimality conditions and Toland's duality for a nonconvex minimization problem," Matematichki Vesnik, vol. 55, no. 1-2, pp. 21-30, 2003.

[3] R. I. Boț, S.-M. Grad, and G. Wanka, "Generalized MoreauRockafellar results for composed convex functions," Optimization, vol. 58, no. 7, pp. 917-933, 2009.

[4] D. H. Fang, C. Li, and K. F. Ng, "Constraint qualifications for extended Farkas's lemmas and Lagrangian dualities in convex infinite programming," SIAM Journal on Optimization, vol. 20, no. 3, pp. 1311-1332, 2009.

[5] R. I. Boţ, Conjugate Duality in Convex Optimization, Springer, Berlin, Germany, 2010.

[6] N. Dinh, T. T. A. Nghia, and G. Vallet, "A closedness condition and its applications to DC programs with convex constraints," Optimization, vol. 59, no. 3-4, pp. 541-560, 2010.
[7] D. H. Fang, C. Li, and X. Q. Yang, "Stable and total Fenchel duality for DC optimization problems in locally convex spaces," SIAM Journal on Optimization, vol. 21, no. 3, pp. 730-760, 2011.

[8] D. H. Fang, C. Li, and X. Q. Yang, "Asymptotic closure condition and Fenchel duality for DC optimization problems in locally convex spaces," Nonlinear Analysis: Theory, Methods \& Applications, vol. 75, no. 8, pp. 3672-3681, 2012.

[9] D. H. Fang, G. M. Lee, C. Li, and J. C. Yao, "Extended Farkas's lemmas and strong Lagrange dualities for DC infinite programming," Journal of Nonlinear and Convex Analysis, vol. 14, no. 4, pp. 747-767, 2013.

[10] X. K. Sun and S. J. Li, "Duality and Farkas-type results for extended Ky Fan inequalities with DC functions," Optimization Letters, vol. 7, no. 3, pp. 499-510, 2013.

[11] X.-K. Sun, S.-J. Li, and D. Zhao, "Duality and Farkas-type results for DC infinite programming with inequality constraints," Taiwanese Journal of Mathematics, vol. 17, no. 4, pp. 1227-1244, 2013.

[12] X.-K. Sun, "Regularity conditions characterizing FenchelLagrange duality and Farkas-type results in DC infinite programming," Journal of Mathematical Analysis and Applications, vol. 414, no. 2, pp. 590-611, 2014.

[13] L. Thibault, "Sequential convex subdifferential calculus and sequential Lagrange multipliers," SIAM Journal on Control and Optimization, vol. 35, no. 4, pp. 1434-1444, 1997.

[14] V. Jeyakumar, G. M. Lee, and N. Dinh, "New sequential Lagrange multiplier conditions characterizing optimality without constraint qualification for convex programs," SIAM Journal on Optimization, vol. 14, no. 2, pp. 534-547, 2003.

[15] V. Jeyakumar, Z. Y. Wu, G. M. Lee, and N. Dinh, "Liberating the subgradient optimality conditions from constraint qualifications," Journal of Global Optimization, vol. 36, no. 1, pp. 127137, 2006.

[16] R. I. Boţ, E. R. Csetnek, and G. Wanka, "Sequential optimality conditions for composed convex optimization problems," Journal of Mathematical Analysis and Applications, vol. 342, no. 2, pp. 1015-1025, 2008.

[17] F. S. Bai, Z. Y. Wu, and D. L. Zhu, "Sequential Lagrange multiplier condition for $\epsilon$-optimal solution in convex programming," Optimization, vol. 57, no. 5, pp. 669-680, 2008.

[18] R. I. Boţ, I. B. Hodrea, and G. Wanka, " $\epsilon$-optimality conditions for composed convex optimization problems," Journal of Approximation Theory, vol. 153, no. 1, pp. 108-121, 2008.

[19] N. Dinh, B. S. Mordukhovich, and T. T. A. Nghia, "Qualification and optimality conditions for DC programs with infinite constraints," Acta Mathematica Vietnamica, vol. 34, no. 1, pp. 123-153, 2009.

[20] N. Dinh, J. J. Strodiot, and V. H. Nguyen, "Duality and optimality conditions for generalized equilibrium problems involving DC functions," Journal of Global Optimization, vol. 48, no. 2, pp. 183-208, 2010.

[21] G. S. Kim and G. M. Lee, "On $\epsilon$-optimality theorems for convex vector optimization problems," Journal of Nonlinear and Convex Analysis, vol. 12, no. 3, pp. 473-482, 2011.

[22] G. M. Lee, G. S. Kim, and N. Dinh, "Optimality conditions for approximate solutions of convex semi-infinite vector optimization problems," in Recent Developments in Vector Optimization, Vector Optimization, Q. H. Ansari and J. C. Yao, Eds., vol. 1, pp. 275-295, Springer, Berlin, Germany, 2012.

[23] X. L. Guo, S. J. Li, and K. L. Teo, "Subdifferential and optimality conditions for the difference of set-valued mappings," Positivity, vol. 16, no. 2, pp. 321-337, 2012. 
[24] X. L. Guo and S. J. Li, "Optimality conditions for vector optimization problems with difference of convex maps," Journal of Optimization Theory and Applications, 2013.

[25] D. H. Fang and X. P. Zhao, "Local and global optimality conditions for DC infinite optimization problems," Taiwanese Journal of Mathematics, 2013.

[26] J. B. Hiriart-Urruty, "From convex optimization to nonconvex optimization necessary and sufficient conditions for global optimality," in From Convexity to Nonconvexity, R. P. Gilbert, P. D. Panagiotopoulos, and P. M. Pardalos, Eds., pp. 219-239, Kluwer, London, UK, 2001. 


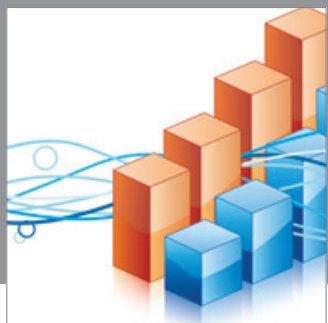

Advances in

Operations Research

mansans

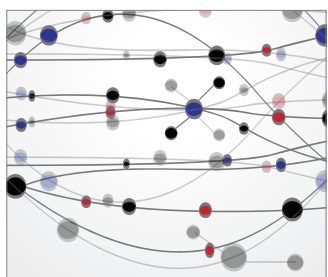

The Scientific World Journal
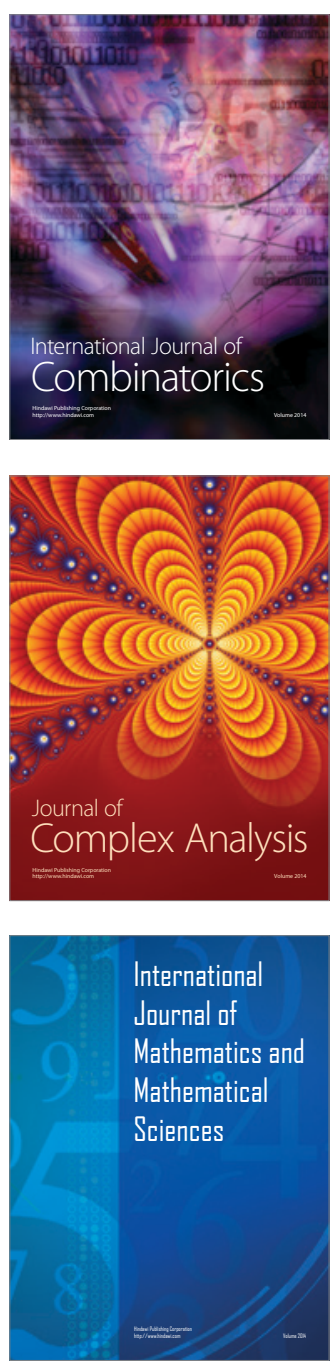
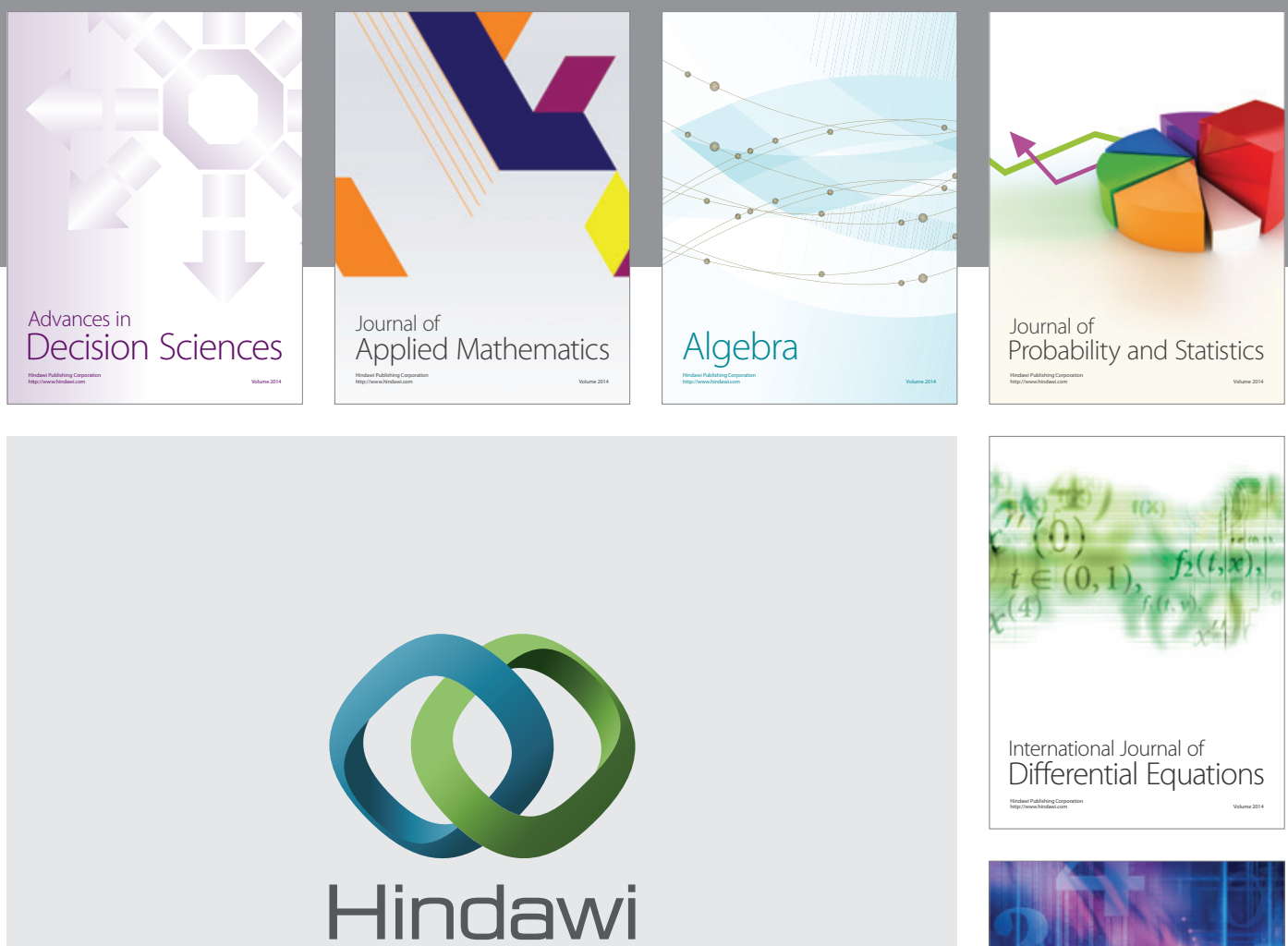

Submit your manuscripts at http://www.hindawi.com
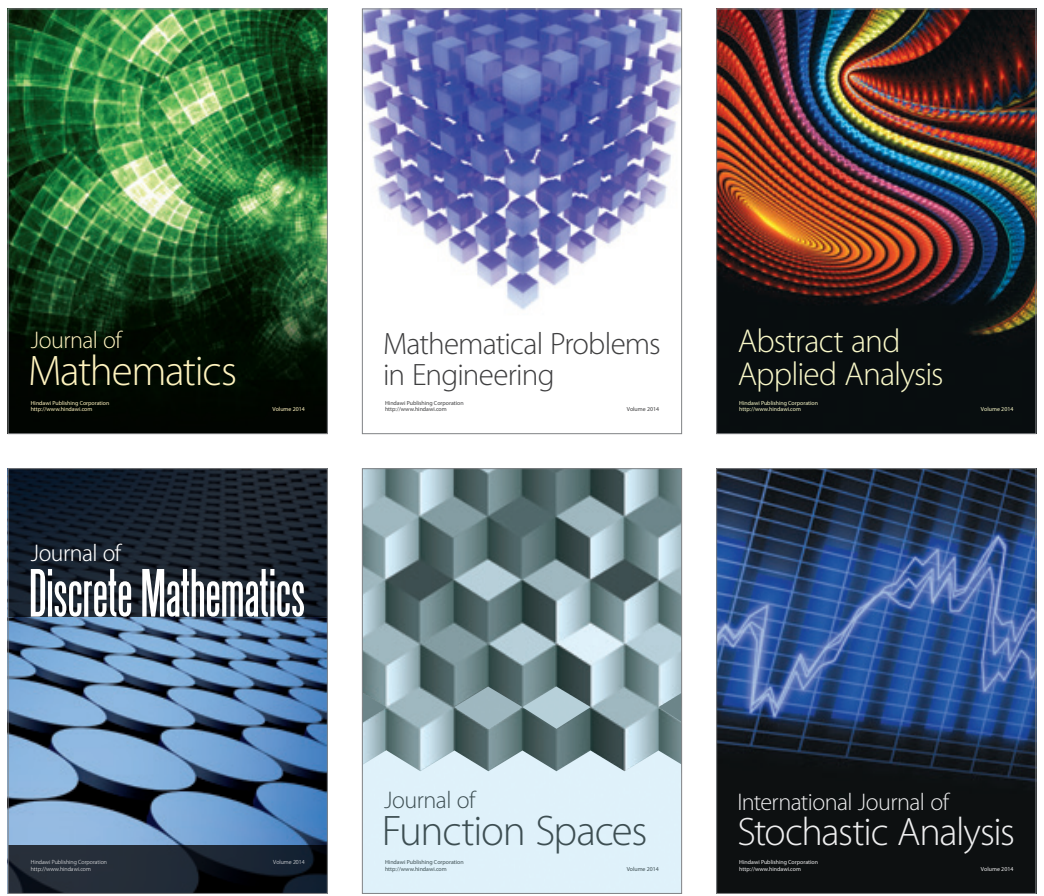

Journal of

Function Spaces

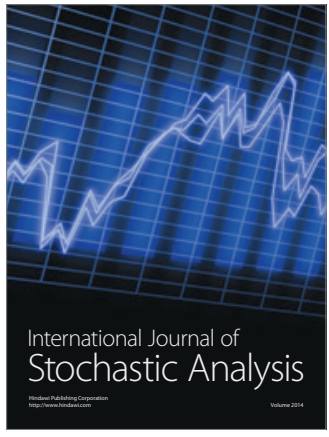

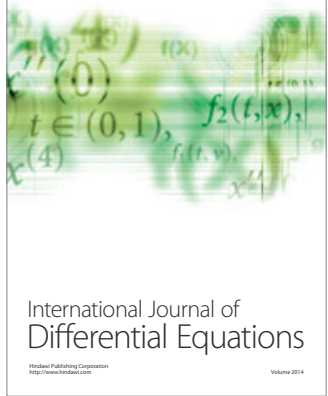
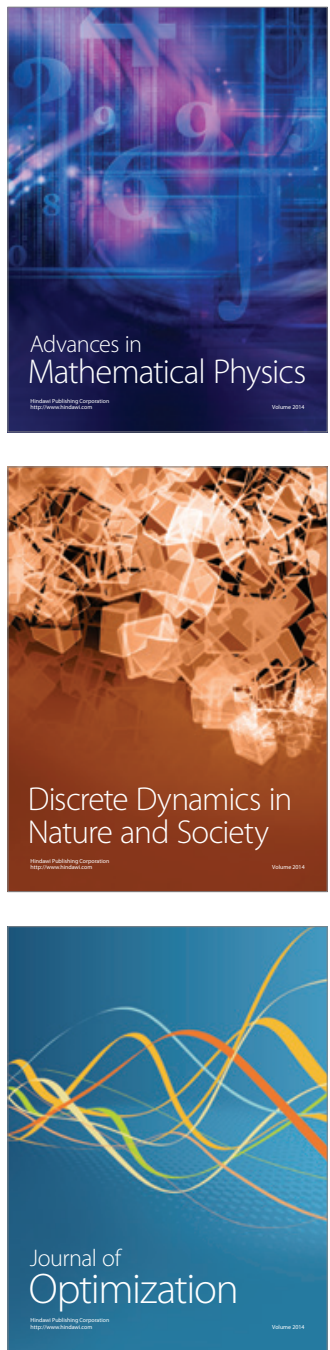